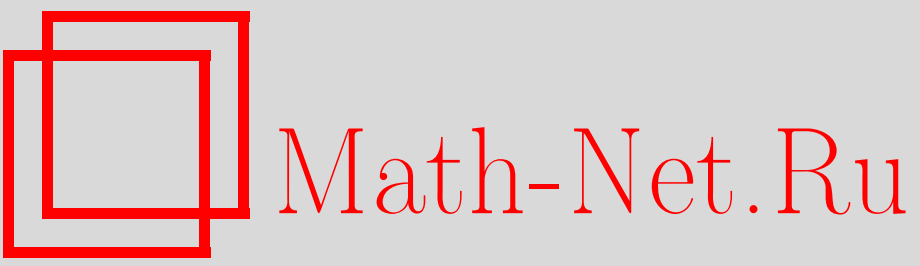

Е. С. Корчак, Математическое моделирование процессов, происходящих в рабочих цилиндрах гидравлического пресса при их декомпрессии, Вестн. Сам. гос. техн. ун-та. Сер. Физ.-мат. науки, 2010, выпуск 5(), 152-158

DOI: https://doi.org/10.14498/vsgtu760

Использование Общероссийского математического портала Math-Net.Ru подразумевает, что вы прочитали и согласны с пользовательским соглашением

http: //www. mathnet.ru/rus/agreement

Параметры загрузки :

IP : 35.173 .219 .12

26 апреля 2023 г., 14:49:21 
УДК 517.958:621.226

\section{МАТЕМАТИЧЕСКОЕ МОДЕЛИРОВАНИЕ ПРОЦЕССОВ, ПРОИСХОДЯЩИХ В РАБОЧИХ ЦИЛИНДРАХ ГИДРАВЛИЧЕСКОГО ПРЕССА ПРИ ИХ ДЕКОМПРЕССИИ}

\section{E. C. Корчак}

Донбасская государственная машиностроительная академия, Украина, 84313, г. Краматорск, ул. Шкадинова, 72.

E-mail: helen_korchak@ukr.net

Рассмотрены основные способы декомпрессии, используемые в современных системах управления, а также математическая модель процессов, происходящих в рабочих иилиндрах гидравлического пресса при их декомпрессии. Дано описание механизма колебаний столба жидкости в силовых иилиндрах. Проанализированы колебательные явления в рабочих иилиндрах при декомпрессии, дано их математическое описание. Численным анализом на базе ковочного гидравлического пресса активным усилием 60 МН показаны особенности типового прочесса декомпрессии рабочих иилиндров. Даны практические рекомендации относительно использования различных разгрузочных клапанов и их влияния на эксплуатационные свойства прессов.

Ключевые слова: декомпрессия, колебания давления, разгрузочный клапан, упругая энергия, рабочий иилиндр, гидравлический пресс.

Введение. Декомпрессия рабочих цилиндров гидравлического пресса осуществляется после того, как в соответствии с технологическим процессом выполнено деформирование заготовки и подвижную поперечину необходимо поднять в исходное верхнее положение. То есть декомпрессия рабочих цилиндров в машинном цикле пресса выполняется в промежутке между окончанием рабочего и началом возвратного хода. В течение этого процесса дросселирующее устройство - дроссельный клапан - должен поглотить суммарную накопленную во время цикла нагружения энергию упругой деформации жидкости и элементов металлоконструкции пресса, превратив ее в тепло. Для достижения наибольшей производительности машины и наилучших режимов декомпрессии системой управления должны быть исключены условия, способствующие возникновению колебательных и ударных явлений в гидроприводе, а также динамических перегрузок частей пресса [1].

1. Механизм процесса декомпрессии рабочих цилиндров гидравлического пресса. Декомпрессия рабочих цилиндров может осуществляться двумя способами [2]:

- применением сливного клапана небольшого сечения, встроенного в главный клапанный распределитель;

- использованием разгрузочного клапана, встроенного в блок наполнительно-сливных клапанов (HCK).

Первому способу соответствует схема, приведённая на рис. 1, а, где открытием сливного клапана 4 осуществляется декомпрессия рабочих цилиндров 1, a HCK 2 открывается только после падения давления в их полостях до $2,5 \div 3,0$ МПа.

Елена Сергеевна Корчак (к.т.н.), старший преподаватель, каф. машин и технологии обработки металлов давлением. 


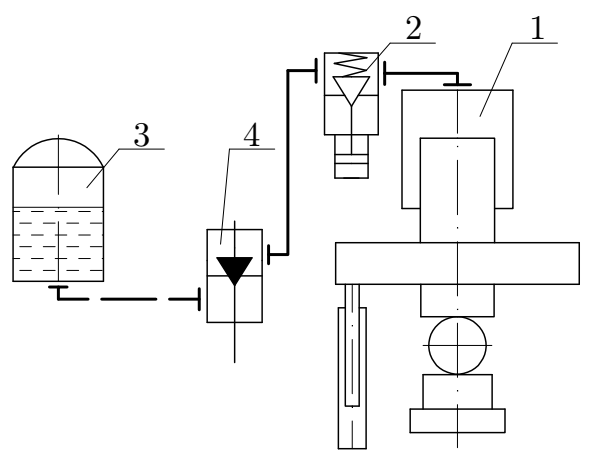

a

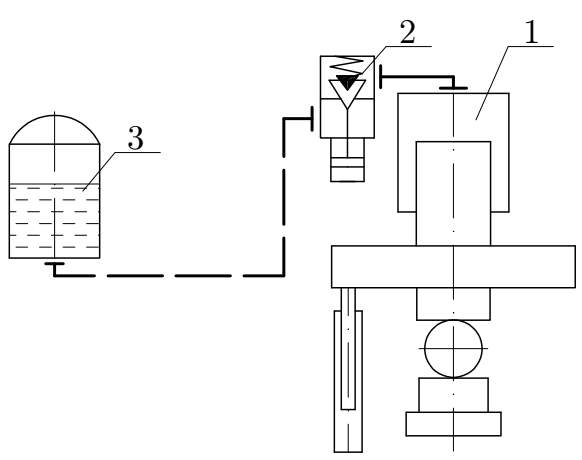

б

Рис. 1. Схемы декомпрессии рабочих цилиндров: 1 - рабочий цилиндр, $2-\mathrm{HCK}, 3-$ сливной бак, 4 - сливной клапан главного клапанного распределителя

Для второго способа (рис. 1, б) характерно то, что сервоцилиндр НCK 2 сначала открывает встроенный в него разгрузочный клапан малого проходного сечения, а затем, после падения давления в рабочих цилиндрах 1 до $2,5 \div 3,0$ МПа, полностью открывается HCK 2 .

Характерным для этих двух способов является длительная задержка при переключении пресса с рабочего на возвратный ход, вызванная повышенным временем декомпрессии полостей рабочих цилиндров от давления из-за необходимости дросселирования рабочей жидкости через малое сечение сливного (рис. 1, a) или разгрузочного (рис. 1, б) клапанов. То есть при этих способах декомпрессии мала интенсивность срабатывания накопленной энергии упругой деформации. Это приводит к тому, что процесс декомпрессии является длительным по времени. Поэтому для определения оптимальных режимов процесса декомпрессии рабочих цилиндров необходимо построить его математическую модель, анализ которой даст возможность уже на стадии проектирования системы управления гидравлическим прессом правильно выбрать параметры и режимы работы разгрузочных устройств.

2. Описание математической модели изменения давления в рабочих цилиндрах. В основу математического описания процесса декомпрессии рабочих цилиндров положено уравнение баланса давлений [3], которое для данной гидравлической системы (рис. 1) можно записать в виде

$$
p_{\text {ц }}-0,5 \rho \xi_{\mathrm{K}} V^{2}-\rho L \frac{d V}{d t}=p_{\delta},
$$

где $p_{\text {ц }}$ - давление в рабочих цилиндрах; $\rho$-плотность рабочей жидкости; $\xi_{\mathrm{k}}$ - приведённый коэффициент гидравлического сопротивления разгрузочного клапана; $V$-скорость движения столба жидкости, находящегося в рабочих цилиндрах; $L$ - приведённая к площади плунжера рабочего цилиндра длина сливного трубопровода; $p_{\delta}$ - давление в наполнительно-сливном баке.

Из анализа уравнения (1) следует, что при истечении жидкости из рабочих цилиндров накопленная энергия упругой деформации расходуется на преодоление гидравлического сопротивления магистрали «рабочие цилиндры - наполнительно-сливной бак» (слагаемое $\left.0,5 \rho \xi_{\mathrm{K}} V^{2}\right)$ и на создание инерционного напора жидкости (слагаемое $\rho L \cdot(d V / d t))$. 
Во время открытия разгрузочного (сливного) клапана его гидравлическое сопротивление изменяется по нижеприведённому закону:

$$
\xi_{\mathrm{K}}=\bar{\zeta}_{\mathrm{K}}\left[1+\alpha\left(\frac{1}{t^{2 n} / t_{\text {отк }}^{2 n}}-1\right)\right],
$$

где $\bar{\zeta}_{\mathrm{K}}-$ коэффициент гидравлического сопротивления полностью открытого клапана; $t_{\text {отк }}$ - время открытия клапана; $n$ - показатель вида конструктивной характеристики клапана; $\alpha$ - коэффициент качества сливной магистрали.

Значение $\bar{\zeta}_{\text {к }}$ можно определить из формулы для расчёта критического проходного сечения $f^{*}$ разгрузочного (сливного) клапана при внезапном расширении струи [4]:

$$
f^{*}=\frac{f_{\mathrm{Tp}}}{\sqrt{\bar{\zeta}_{\mathrm{K}}}+1},
$$

где $f_{\text {тр }}$ - площадь сечения сливного трубопровода.

В окончательном виде математическая модель изменения давления в рабочих цилиндрах пресса в процессе декомпрессии описывается следующим выражением:

$$
\begin{aligned}
& \frac{\rho}{2}\left(\frac{W_{\text {ц }}}{E_{\text {ц }} f_{\text {тр }}}\right)^{2} \bar{\zeta}_{\text {К }}\left[1+\alpha\left(\frac{1}{t^{2 n} / t_{\text {Отк }}^{2 n}}-1\right)\right]\left(\frac{d p_{ц}}{d t}\right)^{2}- \\
&-\rho L \frac{W_{ц}}{E_{ц} f_{\text {тр }}} \frac{d^{2} p_{\text {ц }}}{d t^{2}}+p_{\delta}-p_{\text {ц }}=0,
\end{aligned}
$$

где $E_{ц}$ - приведённый модуль упругости жидкости и металлоконструкции гидравлического пресса; $W_{\text {ц }}$ - объём рабочих цилиндров.

Уравнение (4) описывает изменение давления в рабочих цилиндрах при декомпрессии в статическом виде. Однако переходные процессы в реальных гидравлических системах носят, как правило, колебательный характер [5]. Поскольку колебания в системе декомпрессии рабочих цилиндров мало влияют на закон движения массивных подвижных частей пресса, то нет необходимости рассматривать двух- или трёхмассовую модель «жидкость - подвижные части». Достаточно рассмотреть на основе теории линейных колебаний динамику движения сжимаемой вязкой жидкости в упругом трубопроводе, которая получает ускорение, рассчитанное для «жёсткой» модели гидропривода.

3. Механизм колебаний столба жидкости в рабочих цилиндрах. Представим столб жидкости в рабочем цилиндре (рис. 2) как сложную систему, состоящую из двух элементов [6]: одного 1 повторяющего по конфигурации столб жидкости, но обладающий нулевой массой, и второго 2, расположенного внутри первого, обладающего приведённой массой $m_{\text {пр }}$ и связанного с первым элементом упругой связью в виде пружины.

При декомпрессии система движется вдоль оси $S$ с переменной скоростью $U$ и ускорением $j_{n}$. На рис. 2 последовательно показаны фазы этого движения. Ось $O-O$ - начало отсчета в неподвижной системе координат, ось $O_{1}-O_{1}$ - начало отсчета в подвижной системе координат. 


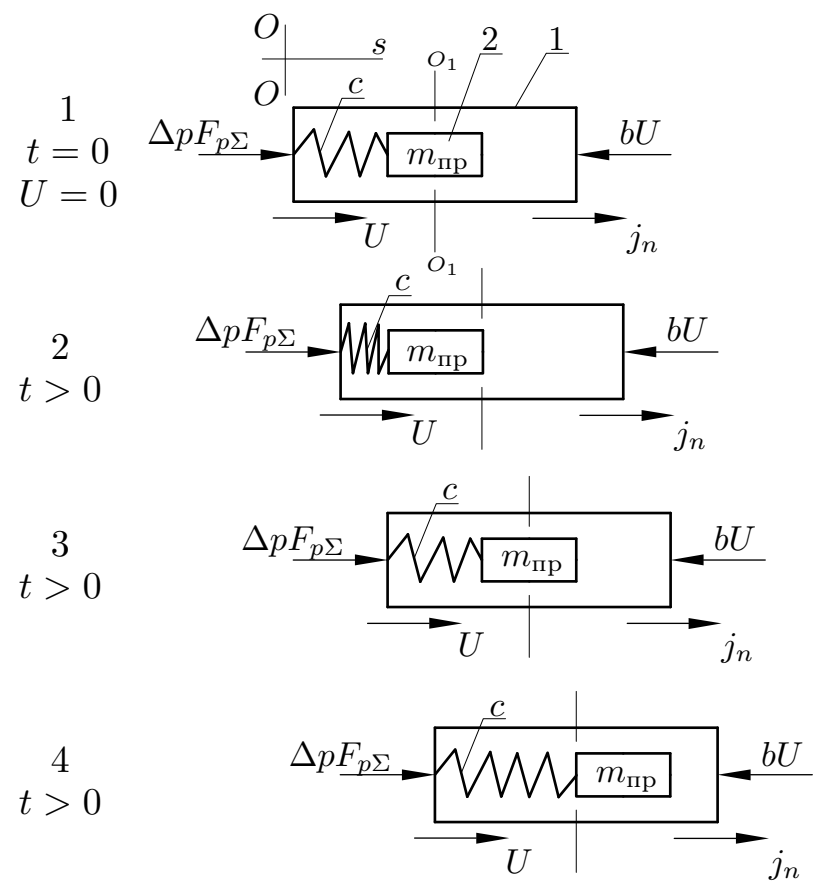

Рис. 2. Колебания столба жидкости в рабочем цилиндре во время декомпрессии

Предположим, что до начала декомпрессии система покоится (рис. 2),

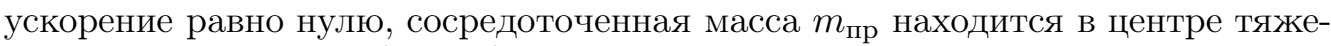
сти столба жидкости (этап 1). В начале декомпрессии элемент 1 под действием силы $\Delta p F_{p \Sigma}$ (где $\Delta p$ - изменение давления в рабочих цилиндрах, $F_{p \Sigma}-$ суммарная площадь рабочих цилиндров) набирает скорость, а второй элемент под действием сил инерции отстаёт от первого и, сжимая пружину, уходит вправо (этап 2). При этом со стороны пружины с жёсткостью $с$ возникает упругая восстанавливающая сила. По мере развития движения упругая сила возрастает настолько, что реверсирует элемент 2 и разгоняет его по направлению движения (этап 3). В момент прохождения элементом 2 начала координат $O_{1}-O_{1}$ упругая сила становится равной нулю, однако за счёт приобретенной кинетической энергии элемент 2 продолжает движение вправо по оси (этап 4). После превращения накопленной кинетической энергии в потенциальную масса $m_{\text {пр }}$ снова реверсируется.

Таким образом, в относительной системе отсчета элемент 2 (приведённая масса столба жидкости) совершает колебательные движения относительно оси $O_{1}-O_{1}$, которые носят затухающий характер, так как колебательному движению препятствует сила вязкого трения $b U$, принимаемая в теории линейных механических колебаний пропорциональной первой степени скорости колебательного движения.

4. Описание математической модели колебаний столба жидкости в рабочих цилиндрах. Основой для построения математической модели является дифференциальное уравнение вынужденных затухающих колебаний приведённой массы столба жидкости в рабочих цилиндрах при сопротивлении, пропорциональном первой степени скорости [7]. Если перейти от колебаний 
столба жидкости к колебаниям давления и выразить ускорение $j_{n}$, то решение дифференциального уравнения можно записать в виде

$$
j_{n}=\frac{\Delta p F_{p \Sigma}}{m_{\text {пр }}[1-\exp (-h t)(\cos \omega t+(h / \omega) \sin \omega t)]},
$$

где $\omega, h$ - параметры колебательной системы.

Выразив из формулы (5) изменение давления $\Delta p$, получим

$$
\Delta p=\frac{j_{n} m_{\text {пр }}}{F_{p \Sigma}}[1-\exp (-h t)(\cos \omega t+(h / \omega) \sin \omega t)] .
$$

Выражение перед квадратными скобками в зависимости (6) представляет собой текущее значение давления в рабочих цилиндрах $p_{\text {ц }}$, определяемое по зависимости (4). Окончательно получим

$$
\Delta p=p_{\text {ц }}[1-\exp (-h t)(\cos \omega t+(h / \omega) \sin \omega t)] .
$$

Выражение (6) является уравнением, связывающим через параметры системы $h$ и $\omega$ ускорение $j_{n}$, соответствующее «жёсткой» модели гидропривода, и изменение давления в системе $\Delta p$ с учётом сжимаемости жидкости и упругости металлоконструкции. Задаваясь на основе паспортных данных пресса допустимым повышением давления на определенном участке гидросистемы и зная параметры уравнения (6) для этого участка, можно определить допустимые ускорения разгона подвижных частей гидравлического пресса.

Параметры $h$ и $\omega$ соответствующих участков гидропривода пресса весьма трудно получить аналитически из-за сложности учёта всех факторов, влияющих на колебательный процесс в жидкости. Поэтому наиболее достоверным является экспериментальный способ их определения методом нанесения единичного возмущения на том или ином участке гидропривода. Затем для каждого участка определяли период колебаний $T$, частоты $\omega=2 \pi / T$, логарифмические декременты затухающих колебаний $\delta=\ln \left(A_{t} / A_{t+T}\right)$ (где $A_{t}$ экспериментальное значение амплитуды колебаний давления) и коэффициент, характеризующий вязкое сопротивление системы $h=\delta / T$.

5. Анализ математической модели процессов, происходящих в рабочих цилиндрах гидравлического пресса при их декомпрессии. Аналитически уравнение (4) не решается. С целью проверки соответствия рассмотренной математической модели реальному процессу декомпрессии её решили численным методом Рунге-Кутта для типового процесса декомпрессии рабочих цилиндров ковочного гидравлического пресса усилием $60 \mathrm{MH}$, работающего на второй ступени усилий $(40 \mathrm{MH})$. Схема процесса представлена на рис. 1 , а. Соответствующие параметры уравнения (4) приведены в таблице.

Результаты решения уравнений (4) (кривая 1) и (7) (кривая 2), а также экспериментальная кривая изменения давления в рабочих цилиндрах при декомпрессии (кривая 3) приведены на рис. 3.

Из полученных графиков (рис. 3) видно, что в течение 0,5 с осуществляется декомпрессия рабочих цилиндров до давления 3,4 МПа через открытый сливной клапан. При этом значения давления, как расчётные (кривая 2), так и экспериментальные (кривая 3), колеблются относительно расчётной кривой 1. Сравнивая расчётные и экспериментальную кривые, можно отметить, 


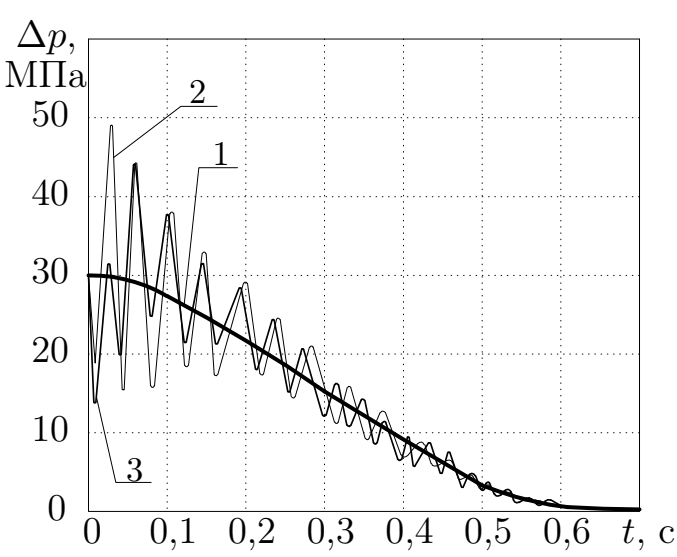

\begin{tabular}{|c|c|c|}
\hline \multicolumn{3}{|c|}{ Параметры уравнения (4) } \\
\hline Параметр & Размерность & Величина \\
\hline $\begin{array}{lll}1 & 1 \\
\end{array}$ & $\mathrm{~K} \Gamma / \mathrm{M}^{3}$ & 1000 \\
\hline$W_{\text {ц }}$ & $\mathrm{M}^{3}$ & 2,92 \\
\hline$E_{\text {Ц }}$ & МПа & 1900 \\
\hline$f_{\text {тр }}$ & $\mathrm{M}^{2}$ & 0,066 \\
\hline $\bar{\zeta}_{\mathrm{K}}$ & - & 45 \\
\hline$\alpha$ & - & 0,8 \\
\hline$t_{\text {отк }}$ & $\mathrm{c}$ & 0,15 \\
\hline$n$ & - & 1,0 \\
\hline$L$ & M & 1320 \\
\hline$p_{\delta}$ & МПа & 0,5 \\
\hline
\end{tabular}

Рис. 3. Изменение давления в рабочих цилиндрах пресса в процессе декомпрессии

что разработанная математическая модель декомпрессии рабочих цилиндров с достаточной степенью достоверности отражает динамику рассматриваемого процесса. Так как декомпрессия осуществлялась сливным клапаном небольшого проходного сечения, встроенным в главный клапанный распределитель, гидроудара в сливной магистрали не наблюдалось.

Заключение. Численным анализом математической модели процессов, происходящих в рабочих цилиндрах гидравлического пресса при их декомпрессии, установлено, что независимо от вида конструктивной характеристики сливного клапана и времени его открытия, а также величины коэффициента $\alpha$, процесс декомпрессии является длительным по времени. Использование сливного клапана с показателем вида конструктивной характеристики $n<1$ приводит к возникновению гидроударных явлений в сливной магистрали, так как такой клапан не обладает необходимыми дросселирующими свойствами и не способен в достаточной мере поглотить накопленную энергию упругой деформации. Замедленный сброс давления из рабочих цилиндров после рабочего хода существенно снижает производительность пресса, затягивает начало движения поперечины вверх, приводит к более интенсивному остыванию заготовки и износу рабочих бойков, что является недопустимым.

Анализ рассмотренной математической модели позволяет сделать вывод, что существующий способ декомпрессии рабочих цилиндров в соответствии со схемами, приведёнными на рис. 1, является неэффективным. Для обеспечения быстрой и безударной декомпрессии необходимо разработать новый механизм осуществления этого процесса.

\section{БИБЛИОГРАФИЧЕСКИЙ СПИСОК}

1. Андронов Г.Ф., Ионкин А.Я., Михайлов А.И. О безударной разгрузке цилиндров гидравлических прессов // Кузнечно-штамповочное производство, 1976. - №10. - С. 3133.

2. Korchak E. S. Processes taking place in power cylinders of hydraulic presses while unloading / In: Research and Development in Mechanical Industry: Papers from the $7^{\text {th }}$ International Conference (RaDMI 2007, 16-20 September 2007, Belgrade, Serbia). — Belgrade, 2007. P. 196-201.

3. Данилов Ю. А., Кирилловский Ю. Л., Колпаков Ю. Г. Аппаратура объёмных гидроприводов: Рабочие процессы и характеристики. - М.: Машиностроение, 1990. - 272 с. 
4. Арзуманов Э. С. Гидравлические регулирующие органы систем автоматического управления. - М.: Машиностроение, 1985. - 256 с.

5. Попов Н.И. Метод оценки влияния сжимаемости жидкости на динамику переходных процессов в гидросистемах прессов // Кузнечно-чтамповочное производство, 1981. № 10 . - C. 25-26.

6. Шинкаренко О. М., Корчак E. С. Процессы, происходящие в рабочих цилиндрах пресса при разгрузке / В сб.: Совершенствование процессов и оборудования обработки давлением в металлургии и машиностроении: Тематич. сб. науч. тр.. - Краматорск: ДГМА, 2007. - C. 294-297.

7. Пановко Я. Г. Основы прикладной теории колебаний и удара. - Л.: Политехника, 1990. $271 \mathrm{c.}$

MSC: 93A30

\section{MATHEMATICAL MODELLING OF PROCESSES OCCURING IN POWER CYLINDERS OF HYDRAULIC PRESS WHILE IT'S DECOMPRESSION}

\section{E. S. Korchak}

Donbass State Engineering Academy,

72, Shkadinova st., Kramatorsk, 84313, Ukraine.

E-mail: helen_korchak@ukr.net

The main ways of decompression used in modern control systems and mathematical modelling of processes occuring in power cylinders under hydraulic press during its decompression are considered. Mechanism of fluid column oscillations in power cylinders is described. Oscillating effects in power cylinders while decompression is analyzed, its mathematical description is given. By means of numerical analysis on the basis of hydraulic forging press with active force of $60 \mathrm{MN}$ peculiarities of typical process of power cylinders decompression are shown. Practical recommendations concerning different decompressing valves using and its influence on maintenance properties of presses are given.

Key words: decompression, pressure oscillations, decompresing valve, elastic energy, power cylinder, hydraulic press.

Original article submitted 18/I/2010; revision submitted 29/IX/2010.

$\overline{\text { Elena S. Korchak (Ph. D. (Tech.)) }}$ Lecturer, Dept. of Machines \& Technology of Plastic Metal Deformation. 